\title{
All-fiber nonlinearity- and dispersion-managed dissipative soliton nanotube mode-locked laser
}

\author{
Z. Zhang,,$^{1,2}$ D. Popa, ${ }^{3, a)}$ V. J. Wittwer, ${ }^{3}$ S. Milana,${ }^{3}$ T. Hasan, ${ }^{3}$ Z. Jiang, ${ }^{3}$ A. C. Ferrari, ${ }^{3}$ \\ and F. O. Ilday 1,4 \\ ${ }^{1}$ Department of Physics, Bilkent University, 06800 Ankara, Turkey \\ ${ }^{2}$ Nanjing University of Posts and Communications, Nanjing 210003, China \\ ${ }^{3}$ Cambridge Graphene Centre, University of Cambridge, Cambridge CB3 OFA, United Kingdom \\ ${ }^{4}$ Department of Electrical and Electronics Engineering, Bilkent University, 06800 Ankara, Turkey
}

(Received 29 September 2015; accepted 27 November 2015; published online 17 December 2015)

\begin{abstract}
We report dissipative soliton generation from an Yb-doped all-fiber nonlinearity- and dispersionmanaged nanotube mode-locked laser. A simple all-fiber ring cavity exploits a photonic crystal fiber for both nonlinearity enhancement and dispersion compensation. The laser generates stable dissipative solitons with large linear chirp in the net normal dispersion regime. Pulses that are 8.7 ps long are externally compressed to $118 \mathrm{fs}$, outperforming current nanotube-based $\mathrm{Yb}$-doped fiber laser designs. (C) 2015 AIP Publishing LLC. [http://dx.doi.org/10.1063/1.4937461]
\end{abstract}

Passively mode-locked fiber lasers are established tools for generating ultrafast pulses due to their compact design, ${ }^{1,2}$ misalignment-free waveguide format, ${ }^{1,2}$ and low cost, ${ }^{1,2}$ while providing a convenient platform for the study of new mode-locking regimes. ${ }^{3-5}$ Mode-locked pulse characteristics are determined by the interaction of dispersion and nonlinear effects. ${ }^{4-6}$ A common approach relies on soliton mode-locking, ${ }^{7}$ where anomalous (negative) dispersion effects are balanced by intensity-dependent nonlinear effects triggered by the pulse itself. ${ }^{8}$ As the net cavity dispersion approaches zero, the laser generates dispersion-managed solitons ${ }^{8}$ or stretched pulses, ${ }^{9}$ with higher pulse energy ranging from hundreds of pJ (Refs. 8 and 9) up to few $\mathrm{nJ},{ }^{10,11}$ than in a soliton laser, where the typical output is in the tens of pJ per pulse range. ${ }^{89}$ Cavities with positive (normal) dispersion, such as similariton ${ }^{3,4}$ or all-normal dispersion lasers, ${ }^{12}$ can produce highly chirped pulses with energies up to tens to hundreds of nJ. ${ }^{12,13}$ These pulses are termed dissipative solitons (DS), ${ }^{5,6}$ with the pulse shaping mechanism based, in part, on spectral filtering of the chirped pulse, which trims the temporal wings of the pulse. ${ }^{12}$ There is great interest in developing DS lasers, ${ }^{6}$ as they can emit stable, high-energy pulses $^{12}$ that can be compressed to transform-limited pulses with numerous applications. ${ }^{14}$ All-fiber cavity designs are preferred for their environmental robustness. A route to enable stable, $100 \mathrm{fs}$ range, DS is to combine both dispersion and nonlinearity management, ${ }^{16}$ especially for lasers operating at $\sim 1 \mu \mathrm{m}$ where standard fiber has normal dispersion only. ${ }^{14}$ In order to provide sufficient dispersion and nonlinear effects, few-kilometer-long cavities have been used, ${ }^{17}$ achieving stable, linearly polarized nanosecond pulses. ${ }^{18}$ However, despite their long, $\sim$ ns range, pulses, such long cavities have repetition rates of hundreds of $\mathrm{kHz}$ (e.g., $1 \mathrm{~km}$ corresponds to $\sim 200 \mathrm{kHz}$ ), ${ }^{17,18}$ often not desirable in applications such as nano-surgery ${ }^{19}$ or two-photon microscopy, ${ }^{20}$ where there is a trade-off between the repetition rate and the multi-photon signal generated. ${ }^{20}$

${ }^{a)}$ Electronic mail: dp387@cam.ac.uk
While nonlinear effects are essential for mode-locking, ${ }^{3,4}$ excessive nonlinearity can result in noise-like or multiple pulse operation. ${ }^{3}$ Nonlinearity management has been proposed as a way of preventing spectral sideband formation in soliton fiber lasers,${ }^{16}$ with consequently higher pulse energy, ${ }^{16}$ while it can be beneficially exploited towards, e.g., similariton generation, ${ }^{3,4}$ spectral broadening, and supercontinuum generation in nonlinear optical fibers. ${ }^{21}$ Similarly, a lack of nonlinearities can also compromise mode-locking formation. For example, management towards increasing nonlinearities was proposed by using quasi-phase-matching devices in freespace solid-state lasers. ${ }^{22}$ Thus, nonlinearity management is an important aspect of laser design, in order to design stable, 100 fs range, mode-locking. ${ }^{16,22}$ Photonic crystal fibers $(\mathrm{PCFs}),{ }^{23}$ i.e., silica fibers with an ordered array of air-holes along their length, ${ }^{23}$ enable advanced dispersion and nonlinearity control in fiber format, ${ }^{23}$ primarily determined by the air-hole spacing, ${ }^{23}$ thus offering possibilities for both nonlinearity and dispersion management in fiber lasers. ${ }^{24,25}$

Carbon nanotubes (CNTs) and graphene have emerged as promising saturable absorbers (SAs) ${ }^{26-28}$ with ultrafast recovery time, ${ }^{29,30}$ able to support short pulses, ${ }^{31,32}$ and with a number of favorable properties for laser development, such as broadband operation, ${ }^{33,34}$ and ease of fabrication ${ }^{27}$ and integration ${ }^{27}$ into all-fiber configurations. ${ }^{35-38}$ Broadband operation is an intrinsic property of graphene, ${ }^{39}$ while in CNTs this can be achieved using a distribution of tube diameters. ${ }^{33}$ A variety of techniques have been implemented in order to integrate CNTs and graphene into lasers. ${ }^{40-42}$ For example, graphene can be integrated into various optical components, with the possibility of controlling the modulation depth. ${ }^{43}$ CNTs and graphene can also be embedded in polymer matrices that can be integrated into fiber lasers. ${ }^{26,27}$ CNT composites can exhibit high ( 20\% (Ref. 32)) modulation depths, ${ }^{32,33}$ preferred for mode-locking of fiber lasers, that typically operate with higher gain and cavity losses ${ }^{2}$ than their solid-state counterparts. ${ }^{44}$ Although CNT modelocked DSs have been reported at $\sim 1.5 \mu \mathrm{m}$ by exploiting dispersion management, ${ }^{15}$ nonlinearity management is also 
required ${ }^{16}$ in order to generate stable, $\sim 100$ fs range DS, even more important for all-fiber lasers operating at $\sim 1 \mu \mathrm{m}$, where the normal dispersion of silica is dominant. ${ }^{14}$

Here, we demonstrate a DS, all-fiber Yb-doped (YDF) laser based on a CNT-SA, operating at $\sim 1040 \mathrm{~nm}$. To retain the all-fiber format, a length of PCF is used for nonlinearity and dispersion management. As a result, DSs are generated in a compact $34-\mathrm{MHz}$ repetition rate laser. Highly chirped, 8.7-ps pulses, are externally compressed in a second-order, $\beta_{2}$, grating pair dispersive delay line, close to their transformlimited duration of $118 \mathrm{fs}$, the shortest to date for CNT YDF DSs fiber lasers. We perform numerical simulations to confirm DS operation.

For our fiber laser design, we use a CNT-SA. To match the operation wavelength of the YDF laser $\left(\sim 1040 \mathrm{~nm},{ }^{45}\right.$ i.e., $\sim 1.19 \mathrm{eV}$ ), CNTs with $\sim 0.8 \mathrm{~nm}$ diameter are required, since this diameter corresponds to a band-gap $\sim 1.18 \mathrm{eV}^{46}$ We use CNTs produced by catalytic disproportionation of $\mathrm{CO}$ $(\mathrm{CoMoCAT}),{ }^{47,48}$ with a $0.6-1.2 \mathrm{~nm}$ diameter distribution. ${ }^{48}$ In order to monitor defects before and after composite fabrication, we measure the Raman spectra of the CoMoCAT powders (red curves) at $633 \mathrm{~nm}$, using a Renishaw InVia microRaman spectrometer equipped with a $100 \mathrm{X}$ objective (N.A. $=0.85$ ). The power is kept below $500 \mu \mathrm{W}$ to avoid damage and laser-induced heating. Fig. 1(a) plots the Raman spectra of the CNT powder in the $\mathrm{G}$ region. The relative intensity of the $D$ to $G$ peaks, $I(D) / I(G)$, can be used to quantify defects. ${ }^{49-51}$ A polymer composite is then fabricated via solution processing, using polyvinyl alcohol (PVA) ${ }^{27}$ The Raman spectra of the CNT-PVA film, Fig. 1(a) (black curve) does not show any increase in $\mathrm{I}(\mathrm{D}) / \mathrm{I}(\mathrm{G})$, indicating that the fabrication process does not induce significant structural damage. Fig. 1(b) plots the absorbance of the PVA (blue line) and the CNT-PVA composite (black line). The latter has a peak between 980 and $1100 \mathrm{~nm}$, which covers the desired operating wavelength, $1040 \mathrm{~nm}$. The absorbance of the PVA is $\sim 1$ order of magnitude lower than that of the CNT-PVA composite, in the $400-1600 \mathrm{~nm}$ range, thus negligible.

For our all-fiber laser, we implement a ring cavity, as shown in Fig. 2(a). The net dispersion is kept normal to support DS. ${ }^{5,6}$ The gain section is a $1.88 \mathrm{~m}$ YDF with $\beta_{2}$ $=35.5 \mathrm{fs}^{2} / \mathrm{mm}$, followed by a $20 \%$ output coupler and an inline isolator. We use a $2.1 \mathrm{~m}$ solid-core PCF for nonlinearity and dispersion management, with an air-hole diameter of $1.2 \mu \mathrm{m}$ and an air-hole spacing of $2.0 \mu \mathrm{m}$, characterised using a scanning electron microscope (SEM) (Fig. 2(b)), corresponding to a nonlinear coefficient of $52 \mathrm{~W}^{-1} \mathrm{~km}^{-1}$ and $\beta_{2}=-46.3 \mathrm{fs}^{2} / \mathrm{mm}^{25}$
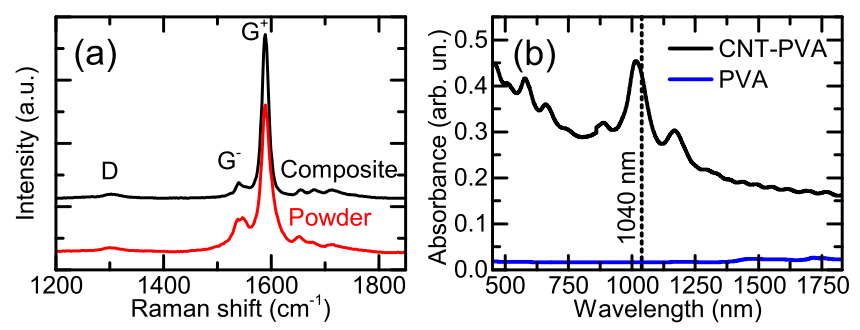

FIG. 1. (a) Raman spectra at $633 \mathrm{~nm}$ for (red lines) powders and (black lines) CNT-PVA in the G peak region. (b) Absorbance of CNT-PVA (black line) and PVA (blue line). Our mode-locked laser operating wavelength is marked by the dotted line.

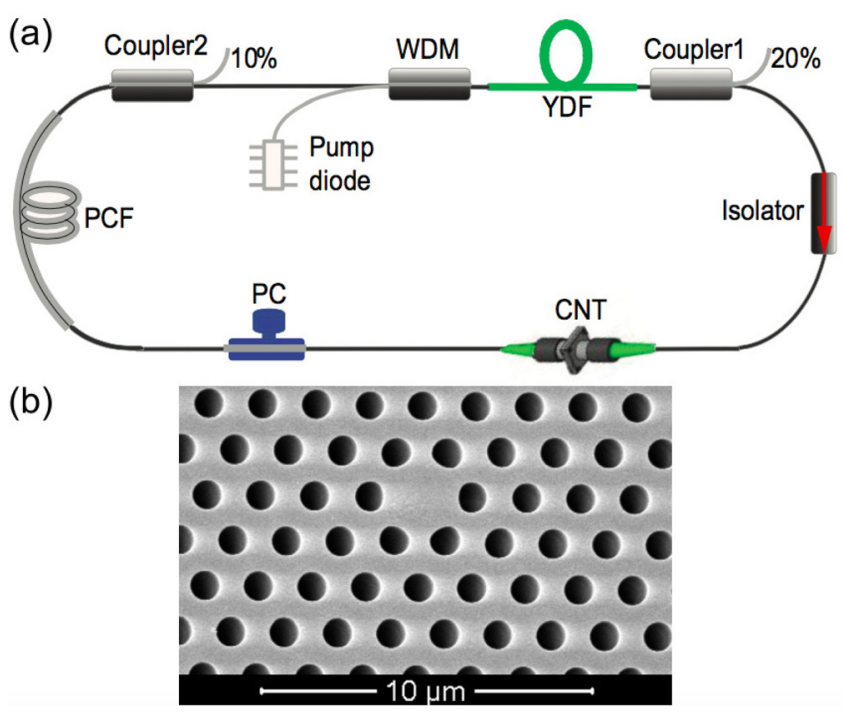

FIG. 2. (a) Schematic of the all-fiber dissipative soliton Yb-doped fiber laser using a CNT-SA and a PCF for nonlinearity and dispersion management. (b) SEM image of the PCF.

All other fibers used in the cavity are standard single-mode fibers (SMF-28 from Corning). The intracavity dispersion is estimated to be $\sim 0.014 \mathrm{ps}^{2}$, comparable to that typically reported for DS lasers. ${ }^{5,6}$ The CNT-SA is then integrated into the cavity between a pair of fiber connectors. ${ }^{27}$ For intracavity polarization adjustment (mode-locking optimization) a polarization controller (PC) is placed after the CNT-SA. Another 10:90 coupler is used to monitor the output before the wavelength-division multiplexer (WDM). The cavity length is $\sim 6 \mathrm{~m}$, corresponding to a repetition rate of $34 \mathrm{MHz}$.

Characterization of mode-locked operation is presented in Fig. 3. Onset of continuous-wave (CW) and mode-locked operations are observed at pump powers $\left(P_{\mathrm{p}}\right)$ of $51 \mathrm{~mW}$ and $74 \mathrm{~mW}$, respectively. A set of mode-locked output spectra is recorded for a constant $P_{\mathrm{p}}=92 \mathrm{~mW}$, and presented in Figs. 3(a) and 3(b) on a logarithmic and linear scale, respectively. The spectrum exhibits steep edges, as a result of spectral filtering, ${ }^{12}$ typical signature of $\mathrm{DS},{ }^{6}$ with $17.6 \mathrm{~nm}$ spectral
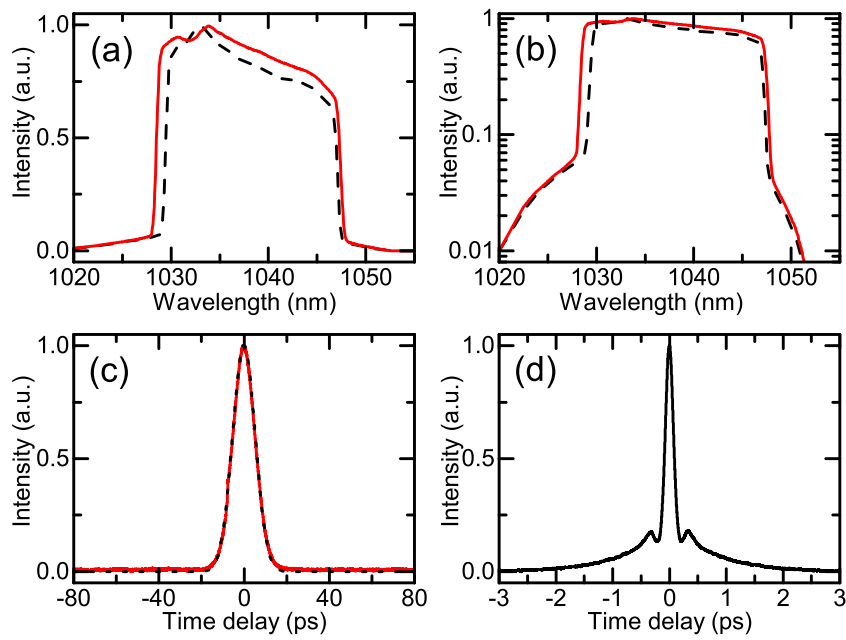

FIG. 3. Optical spectra measured from couplers before (black, dashed line) and after (red, solid line) YDF on (a) linear and (b) logarithmic scale. (c) Autocorrelation trace of the chirped pulse (red, solid line) and its Gaussian fit (black, dashed line). (d) Autocorrelation trace of the dechirped pulse. 
width. The corresponding autocorrelation trace of the chirpedpulse is presented in Fig. 3(c). Assuming a Gaussian profile, this gives a pulse duration of $8.7 \mathrm{ps}$. The time-bandwidth product (TBP) is $\sim 100$ times the expected transform-limit value of $0.44 .^{8}$ Using a grating pair, with anomalous dispersion $-0.25 \mathrm{ps}^{2}$, seed pulses are de-chirped to 118 fs (Fig. 3(d)). The resulting TBP is $\sim 0.57$, close to the transform-limit, indicating that the chirp is predominantly linear. After de-chirping, an increased pedestal is observed, likely due to uncompensated dispersion and nonlinearities associated with the pulse propagation in the $\mathrm{PCF}^{25}$

In order to better understand the mode-locking dynamics, we perform numerical simulations based on a modified nonlinear Schrödinger equation model, described in Ref. 4. The evolution of the temporal and spectral widths along the cavity is shown in Fig. 4(a). The pulse duration and spectral width increase monotonously in the YDF and the following SMF segments, which have normal dispersion. As expected, the pulse duration decreases in the PCF segment due to its anomalous dispersion, and increases back slightly in the following SMF segment. The spectral width also increases in the PCF segment due to self-phase modulation. Fig. 4(b) plots the output spectra after the YDF and before the WDM. Their bandwidths are 17.3 and $17.1 \mathrm{~nm}$, respectively. Fig. 4(c) shows a 7.2 ps-long pulse after the YDF. The overall evolution indicates that pulses remain highly chirped throughout the cavity, and temporal breathing is much smaller than in traditional dispersion-managed lasers. ${ }^{8}$ The accumulated nonlinear phase shift in the PCF is estimated to be $0.86 \pi$. This should be contrasted to the case of no dispersion or nonlinearity management, as would be obtained by replacing the PCF with regular SMF of equal length: the resulting nonlinear phase shift is $0.13 \pi$, which is 7 times smaller. However, in such configuration mode-locking could never be initiated.

We then consider the effect of dispersion on the DS operation. We change the SMF length to vary the dispersion. We achieve stable operation with steep-edge spectral shape within a $0.11-0.026 \mathrm{ps}^{2}$ dispersion range. The laser exhibits
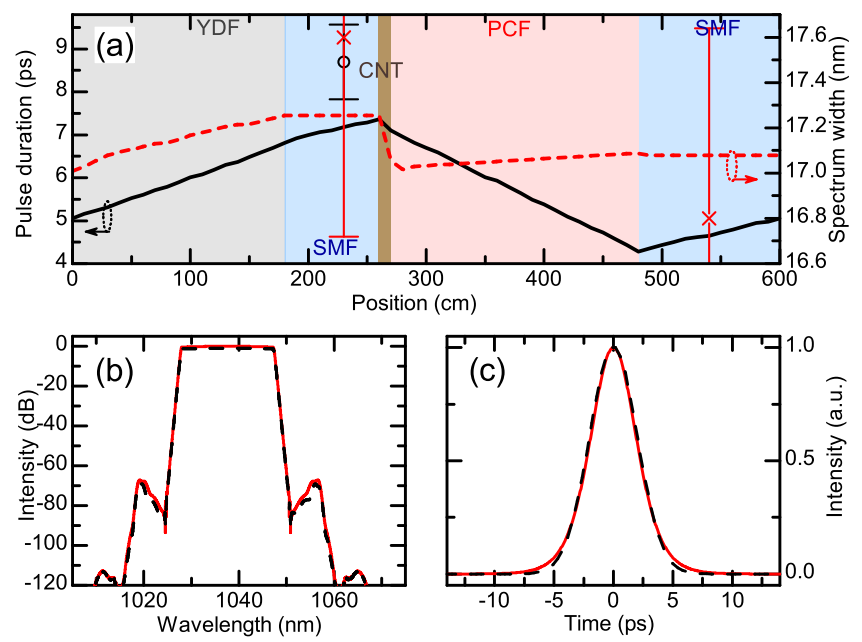

FIG. 4. Simulations. (a) Evolution of the temporal and spectral widths as a function of position along the cavity. (b) Spectra after the YDF (red, solid line) and before the WDM (black, dashed line). (c) Pulse shape after the YDF (red, solid line) and its Gaussian fit (black, dashed line).
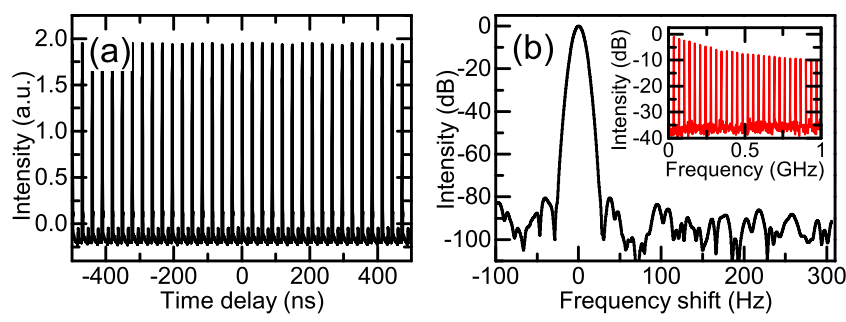

FIG. 5. (a) Output pulse train. (b) RF spectrum with $500 \mathrm{~Hz}$ span and $1 \mathrm{~Hz}$ resolution with central frequency shifted to zero for clarity, inset shows the RF harmonics over a scan range of $1 \mathrm{GHz}$, with $500 \mathrm{kHz}$ resolution bandwidth.

Q-switching with no spectral steep-edge signature outside this range, showcasing the importance of dispersion management for stable DS formation. The spectral width broadens with pump power. The maximum output power is $\sim 3.5 \mathrm{~mW}$, for a pump power of $\sim 100 \mathrm{~mW}$, corresponding to an intracavity pulse energy of $0.5 \mathrm{~nJ}$. Even broader spectral width, combined with shorter pulse duration and higher output power, may be achieved by further improvement of the cavity design. For example, by employing evanescent-field interaction, the CNT-SA would be exposed to only a fraction of the field, with the interaction length extended up to $\mathrm{mm}$, thus avoiding high optical densities for higher-power pumping designs.

The laser stability is characterized through radio frequency (RF) measurements. ${ }^{52}$ Fig. 5 (b) plots the RF spectrum around the fundamental cavity round-trip frequency of 34 $\mathrm{MHz}$ with $1-\mathrm{Hz}$ resolution bandwidth and $500-\mathrm{Hz}$ scanning range. An $80 \mathrm{~dB}$ signal-to-noise ratio is observed, indicating low amplitude fluctuations. There is no spectral modulation over $1 \mathrm{GHz}$ [see inset of Fig. 5(b)], which, when combined with long-range autocorrelation measurements, rules out multiple pulsing.

In conclusion, we reported the generation of DS from an all-fiber laser. The use of a PCF, for both nonlinearity and dispersion management, results in stable, self-starting modelocking, with large linear chirp. Nonlinearity management enables mode-locking at relatively low pump power. The output pulses can be externally compressed to 118 fs. Given the high repetition rate, short pulses generated from an environmentally robust, all-fiber cavity architecture. Our results might be used in a variety of applications, such as nonlinear imaging, nano-surgery, or spectroscopy.

We acknowledge funding from TÜBITTAK Grant No. 113F319, from ERC Grants ERC-617521 NLL and Hetero2D, EPSRC Grant Nos. EP/K01711X/1, EP/K017144/1, and EP/L016087/1, and a Royal Society Wolfson Research Merit Award, the Royal Academy of Engineering and Emmanuel College, Cambridge.

\footnotetext{
${ }^{1}$ M. E. Fermann and I. Hartl, Nat. Photonics 7, 868 (2013).

${ }^{2}$ O. Okhotnikov, Fiber Lasers (Wiley-VCH, Berlin, 2012).

${ }^{3}$ F. Ö. Ilday, J. R. Buckley, W. G. Clark, and F. W. Wise, Phys. Rev. Lett. 92, 213902 (2004).

${ }^{4}$ B. Oktem, C. Ülgüdür, and F. Ö. Ilday, Nat. Photonics 4, 307 (2010).

${ }^{5}$ W. H. Renninger, A. Chong, and F. W. Wise, Phys. Rev. A 77, 023814 (2008).

${ }^{6}$ P. Grelu and N. Akhmediev, Nat. Photonics 6, 84 (2012).

${ }^{7}$ I. N. Duling III, Electron. Lett. 27, 544 (1991).
} 
${ }^{8}$ L. E. Nelson, D. J. Jones, K. Tamura, H. A. Haus, and E. P. Ippen, Appl. Phys. B 65, 277 (1997).

${ }^{9}$ K. Tamura, E. P. Ippen, H. A. Haus, and L. E. Nelson, Opt. Lett. 18, 1080 (1993).

${ }^{10}$ F. Ö. Ilday, F. W. Wise, and T. Sosnowski, Opt. Lett. 27, 1531 (2002).

${ }^{11}$ F. Ö. Ilday, J. R. Buckley, H. Lim, F. W. Wise, and W. G. Clark, Opt. Lett. 28, 1365 (2003).

${ }^{12}$ A. Chong, J. Buckley, W. Renninger, and F. W. Wise, Opt. Express 14, 10095 (2006).

${ }^{13}$ S. Lefrançois, K. Kieu, Y. Deng, J. D. Kafka, and F. W. Wise, Opt. Lett. 35, 1569 (2010).

${ }^{14}$ M. E. Fermann, A. Galvanauskas, and G. Sucha, Ultrafast Lasers: Technology and Applications (CRC Press, 2003).

${ }^{15}$ Z. Zhang, L. Wang, and Y. J. Wang, J. Lightwave Technol. 31, 3719 (2013).

${ }^{16}$ F. Ö. Ilday and F. W. Wise, J. Opt. Soc. Am. B 19, 470 (2002).

${ }^{17}$ E. J. R. Kelleher, J. C. Travers, Z. Sun, A. G. Rozhin, A. C. Ferrari, S. V. Popov, and J. R. Taylor, Appl. Phys. Lett. 95, 111108 (2009).

${ }^{18}$ R. I. Woodward, E. J. R. Kelleher, D. Popa, T. Hasan, F. Bonaccorso, A. C. Ferrari, S. V. Popov, and J. R. Taylor, IEEE Photonic Technol. Lett. 26, 1672 (2014).

${ }^{19}$ S. Yava, M. Erdogan, K. Grel, F. Ö. Ilday, Y. B. Eldeniz, and U. H. Tazebay, Biomed. Opt. Express 3, 605 (2012).

${ }^{20}$ J. Bewersdorf and S. W. Hell, J. Microsc. 191, 28 (1998).

${ }^{21}$ J. M. Dudley and J. R. Taylor, Nat. Photonics 3, 85 (2009).

${ }^{22}$ C. R. Phillips, A. S. Mayer, A. Klenner, and U. Keller, Optica 2, 667 (2015).

${ }^{23}$ P. Russell, Science 299, 358 (2003).

${ }^{24}$ H. Lim, F. Ö. Ilday, and F. Wise, Opt. Express 10, 1497 (2002).

${ }^{25}$ Z. Zhang, Ç. Şenel, R. Hamid, and F. Ö. Ilday, Opt. Lett. 38, 956 (2013).

${ }^{26}$ V. Scardaci, Z. P. Sun, F. Wang, A. G. Rozhin, T. Hasan, F. Hennrich, I. H. White, W. I. Milne, and A. C. Ferrari, Adv. Mater. 20, 4040 (2008).

${ }^{27}$ T. Hasan, Z. Sun, F. Wang, F. Bonaccorso, P. H. Tan, A. G. Rozhin, and A. C. Ferrari, Adv. Mater. 21, 3874 (2009).

${ }^{28}$ Z. Sun, T. Hasan, F. Torrisi, D. Popa, G. Privitera, F. Wang, F. Bonaccorso, D. M. Basko, and A. C. Ferrari, ACS Nano 4, 803 (2010).

${ }^{29}$ J. S. Lauret, C. Voisin, G. Cassabois, C. Delalande, P. Roussignol, O. Jost, and L. Capes, Phys. Rev. Lett. 90, 057404 (2003).

${ }^{30}$ D. Brida, A. Tomadin, C. Manzoni, Y. J. Kim, A. Lombardo, S. Milana, R. R. Nair, K. S. Novoselov, A. C. Ferrari, G. Cerullo, and M. Polini, Nat. Commun. 4, 1987 (2013).

${ }^{31}$ D. Popa, Z. Sun, F. Torrisi, T. Hasan, F. Wang, and A. C. Ferrari, Appl. Phys. Lett. 97, 203106 (2010).

${ }^{32}$ D. Popa, Z. Sun, T. Hasan, W. B. Cho, F. Wang, F. Torrisi, and A. C. Ferrari, Appl. Phys. Lett. 101, 153107 (2012).
${ }^{33}$ F. Wang, A. G. Rozhin, V. Scardaci, Z. Sun, F. Hennrich, I. H. White, W. I. Milne, and A. C. Ferrari, Nat. Nanotechnol. 3, 738 (2008).

${ }^{34}$ R. Going, D. Popa, F. Torrisi, Z. Sun, T. Hasan, F. Wang, and A. C. Ferrari, Physica E 44, 1078 (2012).

${ }^{35}$ M. Zhang, E. J. R. Kelleher, T. H. Runcorn, V. M. Mashinsky, O. I. Medvedkov, E. M. Dianov, D. Popa, S. Milana, T. Hasan, Z. Sun, F. Bonaccorso, Z. Jiang, E. Flahaut, B. H. Chapman, A. C. Ferrari, S. V. Popov, and J. R. Taylor, Opt. Express 21, 23261 (2013).

${ }^{36}$ C. E. S. Castellani, E. J. R. Kelleher, D. Popa, T. Hasan, Z. Sun, A. C. Ferrari, S. V. Popov, and J. R. Taylor, Laser Phys. Lett. 10, 015101 (2013).

${ }^{37}$ D. G. Purdie, D. Popa, V. J. Wittwer, Z. Jiang, G. Bonacchini, F. Torrisi, S. Milana, E. Lidorikis, and A. C. Ferrari, Appl. Phys. Lett. 106, 253101 (2015).

${ }^{38}$ R. I. Woodward, E. J. R. Kelleher, T. H. Runcorn, S. Loranger, D. Popa, V. J. Wittwer, A. C. Ferrari, S. V. Popov, R. Kashyap, and J. R. Taylor, Opt. Lett. 40, 387 (2015).

${ }^{39}$ F. Bonaccorso, Z. Sun, T. Hasan, and A. C. Ferrari, Nat. Photon. 4, 611 (2010).

${ }^{40}$ R. Mary, G. Brown, S. J. Beecher, F. Torrisi, S. Milana, D. Popa, T. Hasan, Z. Sun, E. Lidorikis, S. Ohara, A. C. Ferrari, and A. K. Kar, Opt. Express 21, 7943 (2013).

${ }^{41}$ K. Kieu and F. W. Wise, Opt. Express 16, 11453 (2008).

${ }^{42}$ R. Mary, G. Brown, S. J. Beecher, R. R. Thomson, D. Popa, Z. Sun, F. Torrisi, T. Hasan, S. Milana, F. Bonaccorso, A. C. Ferrari, and A. K. Kar, Appl. Phys. Lett. 103, 221117 (2013).

${ }^{43}$ C. A. Zaugg, Z. Sun, V. J. Wittwer, D. Popa, S. Milana, T. S. Kulmala, R. S. Sundaram, M. Mangold, O. D. Sieber, M. Golling, Y. Lee, J. H. Ahn, A. C. Ferrari, and U. Keller, Opt. Express 21, 31548 (2013).

${ }^{44}$ W. Koechner, Solid-State Laser Engineering (Springer, 1999).

${ }^{45}$ M. J. F. Digonnet, Rare Earth Doped Fiber Lasers and Amplifiers (Marcel Dekker, USA, 1993).

${ }^{46}$ R. B. Weisman and S. M. Bachilo, Nano Lett. 3, 1235 (2003).

${ }^{47}$ D. E. Resasco, W. E. Alvarez, F. Pompeo, L. Balzano, J. E. Herrera, B. Kitiyanan, and A. Borgna, J. Nanopart. Res. 4, 131 (2002).

${ }^{48}$ B. Kitiyanan, W. E. Alvarez, J. H. Harwell, and D. E. Resasco, Chem. Phys. Lett. 317, 497 (2000).

${ }^{49}$ A. C. Ferrari and J. Robertson, Phys. Rev. B 61, 14095 (2000).

${ }^{50}$ A. C. Ferrari and D. M. Basko, Nat. Nanotechnol. 8, 235 (2013).

${ }^{51}$ L. G. Cançado, A. Jorio, E. H. M. Ferreira, F. Stavale, C. A. Achete, R. B. Capaz, M. V. O. Moutinho, A. Lombardo, T. S. Kulmala, and A. C. Ferrari, Nano Lett. 11, 3190 (2011).

${ }^{52}$ D. V. D. Linde, Appl. Phys. B 39, 201 (1986). 